\title{
Large isotropic negative thermal expansion above a structural quantum phase transition
}

\author{
Sahan U. Handunkanda, ${ }^{1}$ Erin B. Curry, ${ }^{1}$ Vladimir Voronov, ${ }^{2}$ Ayman H. Said, ${ }^{3}$ Gian G. Guzmán-Verri, ${ }^{4,5}$ \\ Richard T. Brierley, ${ }^{6}$ Peter B. Littlewood, ${ }^{7,8}$ and Jason N. Hancock ${ }^{1, *}$ \\ ${ }^{1}$ Department of Physics and Institute for Materials Science, University of Connecticut, Storrs, Connecticut 06269, USA \\ ${ }^{2}$ Kirensky Institute of Physics, Krasnoyarsk 660036 Russia \\ ${ }^{3}$ Advanced Photon Source, Argonne National Laboratory, Argonne, Illinois 60439, USA \\ ${ }^{4}$ Centro de Investigación en Ciencia e Ingeniería de Materiales y Escuela de Física, Universidad de Costa Rica, San José 2060, Costa Rica \\ ${ }^{5}$ Materials Science Division, Argonne National Laboratory, Argonne, Illinois 60349, USA \\ ${ }^{6}$ Department of Physics, Yale University, New Haven, Connecticut 06520, USA \\ ${ }^{7}$ James Franck Institute, University of Chicago, Chicago, Illinois 60637, USA \\ ${ }^{8}$ Argonne National Laboratory, Argonne, Illinois 60349, USA
}

(Received 16 June 2015; revised manuscript received 17 August 2015; published 1 October 2015)

\begin{abstract}
Perovskite structured materials contain myriad tunable ordered phases of electronic and magnetic origin with proven technological importance and strong promise for a variety of energy solutions. An always-contributing influence beneath these cooperative and competing interactions is the lattice, whose physics may be obscured in complex perovskites by the many coupled degrees of freedom, which makes these systems interesting. Here, we report signatures of an approach to a quantum phase transition very near the ground state of the nonmagnetic, ionic insulating, simple cubic perovskite material $\mathrm{ScF}_{3}$, and show that its physical properties are strongly effected as much as $100 \mathrm{~K}$ above the putative transition. Spatial and temporal correlations in the high-symmetry cubic phase determined using energy- and momentum-resolved inelastic $\mathrm{x}$-ray scattering as well as $\mathrm{x}$-ray diffraction reveal that soft mode, central peak, and thermal expansion phenomena are all strongly influenced by the transition.
\end{abstract}

DOI: 10.1103/PhysRevB.92.134101 PACS number(s): 64.70.Tg, 05.30.Rt, 62.20.D-, 65.40.De

The class of materials with the perovskite structure and chemical formula $A B X_{3}$ contains examples of perhaps every possible type of physical behavior [1,2], much of which is difficult to understand because of the shear complexity of matter. A rich terrain of structural transitions associated with $B X_{6}$ octahedral tilting in perovskites strongly effects electronic conduction and magnetic exchange pathways, defining the framework of interactions governing a range of physical properties. The $A$-site tolerance appears to be an important parameter in determining the structural phase stability [1-3], but stable $A$-site-free perovskite structures are also thermodynamically stable. These are rare cases among oxides $(X=\mathrm{O})$ because the $B$ ions must take on rare hexavalent $(+6)$ electronic configurations, and the only known instance is $\mathrm{ReO}_{3}$. In perovskites based on fluorine $(X=\mathrm{F})$, however, the $B$ ions assume the common trivalent $(+3)$ configuration in an expanded suite of $A$-site-free perovskite lattices.

Figure 1(a) shows a structural phase diagram of $B F_{3}$ perovskites, where $B$ is a trivalent metal ion [4]. The $3 d$ metal trifluorides display a reversible [5] structural cubic-torhombohedral $(c-r)$ phase boundary. This sequence of $3 d$ transition metal trifluoride compounds is rhombohedral at room temperature, with the exception of $B=\mathrm{Sc}$, which appears at the zero-temperature terminated $c-r$ phase boundary. Indeed, no rhombohedral phase transition has been observed for $\mathrm{ScF}_{3}$ down to $0.4 \mathrm{~K}[6]$, suggesting that near this composition, the structural phase can be driven by a parameter other than temperature, implying that the ground state of this ionic insulator is very near a quantum phase transition (QPT). Cubic $\mathrm{ScF}_{3}$ further stands out among substances in that it has the most stable structural phase of any known solid trifluoride,

\footnotetext{
*Corresponding author: jason.hancock@uconn.edu
}

retaining high cubic symmetry and a four-atom unit cell up to its high melting point, $>1800 \mathrm{~K}[6,7]$. Separate from the QPT reported here, further interest in this system is due to the recent discovery of an extremely large volume negative thermal expansion (NTE) $\left[\alpha_{V} \simeq-34 \times 10^{-6} / \mathrm{K}\right.$ near $300 \mathrm{~K}$, Fig. 2(a)] which is both isotropic and thermally persistent from cryogenic $(20 \mathrm{~K})$ to oven $(1100 \mathrm{~K})$ temperatures [7].

In order to explore this unusual structural quantum material, we have investigated structure and lattice dynamics in high-quality single-crystalline $\mathrm{ScF}_{3}$ using $\mathrm{x}$-ray diffraction and inelastic $\mathrm{x}$-ray scattering (IXS). The high momentum resolution $\left(0.02 \AA^{-1}\right)$, nine-analyzer collection scheme, and microfocused $x$-ray beam permits measurements of the dynamical structure factor [12] within a transparent, isotopically pure, single-crystalline grain $[13,14]$ of mosaic width $\simeq 0.002^{\circ}$, as measured on the strong $(H, K, L)=(1,1,0)$ Bragg reflection indexed to the four-atom simple cubic cell (lattice parameter $a=4.016 \AA$ at temperature $T=300 \mathrm{~K}$ ). The dynamical structure factor we have measured is a fundamental property which contains information on the spatial and temporal fluctuations of the lattice degrees of freedom in $\mathrm{ScF}_{3}$ on the approach to the structural quantum phase transition. Figure 2(b) shows an overview of the lowest-energy mode dispersion in $\mathrm{ScF}_{3}$ along high-symmetry directions determined by fitting the structure factor to a damped harmonic oscillator model (see the Supplemental Material [15]). Along the cut from the Brillouin zone (BZ) face center $X$ to the BZ edge center $M$, an optic mode crosses the acoustic branches and softens dramatically to a low energy of $3.5 \mathrm{meV}$ at room temperature. Cooling the sample to $8 \mathrm{~K}$ results in a dramatic and uniform softening of this entire $M-R$ branch to $\sim 1 \mathrm{meV}$, further suggesting an approach to a structural instability near zero temperature.

The low $M-R$ branch is a near-degenerate manifold of cooperative vibrational modes which are soft because they 


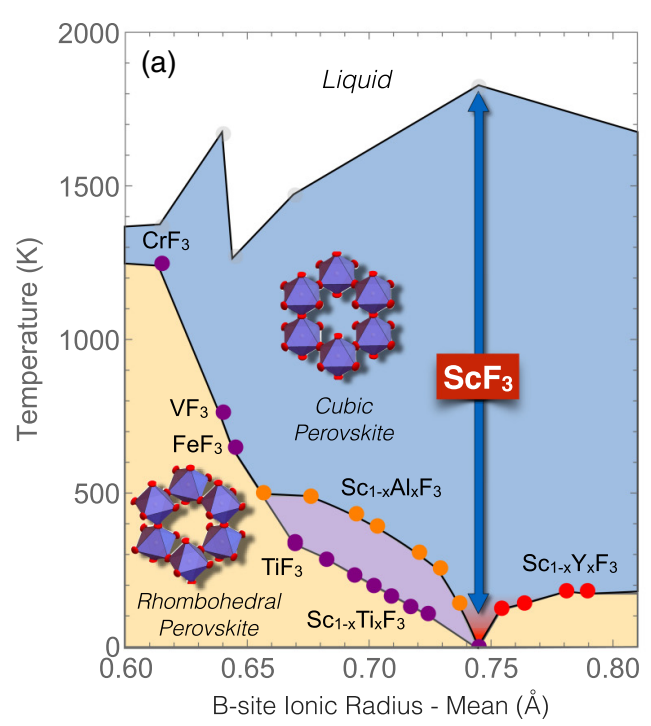

(b)
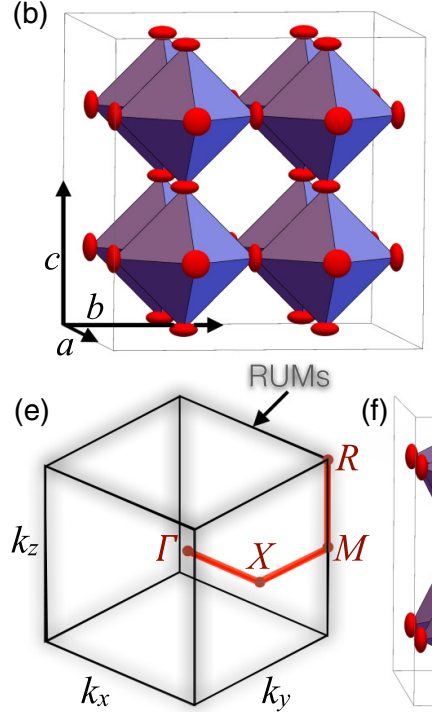

(c)

(f)

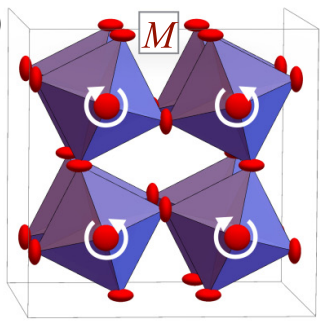

(d)

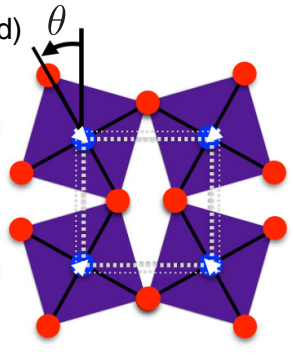

(g)

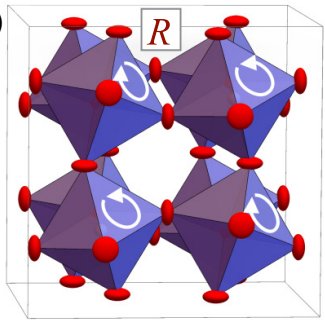

FIG. 1. (Color online) Background material: (a) Structural phase diagram showing the $c-r$ phase boundary vs the $B$-site mean radius in $3 d$ transition metal trifluorides and in the solid solutions $\mathrm{Sc}_{1-x} \mathrm{Al}_{x} \mathrm{~F}_{3}$ and $\mathrm{Sc}_{1-x} \mathrm{Y}_{x} \mathrm{~F}_{3}$. Data taken from Refs. [5,8-11]. (b) Simple cubic structure of $\mathrm{ReO}_{3}, \mathrm{ScF}_{3}$, and the high-temperature phases of transition metal trifluorides. $\mathrm{Sc}^{+3}$ ions sit at the center of regular corner-linked octahedral cages formed by $\mathrm{F}^{-}$ions (red ellipsoids). (c), (d) 100 planar section of the octahedra, illustrating the displacement pattern of modes attributed to NTE. The area of the box in (d) is reduced by $\cos ^{2} \theta$ of the area in (c). (e) The first Brillouin zone of the simple cubic structure, showing the zone center $\Gamma(0,0,0)$, zone face center $X(\pi, 0,0)$, zone edge center $M(\pi, \pi, 0)$, and zone corner $R(\pi, \pi, \pi)$ points. Soft collective rigid unit modes (RUMs) which nearly preserve internal octahedral bonds are permitted on the zone boundaries, as indicated by shadowing. The lowest vibrational modes at the (f) $M$ and (g) $R$ points involve coordinated staggered rotations of octahedra in the patterns shown. The rhombohedral phase can be described as a static tilt according to the $R_{4}^{+}$pattern.

largely preserve the internal dimensions of the stiff metal-anion molecular subunits. In order to retain local constraints of bond distances and angles, staggered rotations induce a shrinking of the cell dimensions [Figs. 1(c) and 1(d) show this for the $M_{3}^{+}$distortion], establishing a long-range coupling between local transverse linkage fluctuations and lattice volume that has long been ascribed as the cause of structural NTE [16-19]. $M-R$ is an important mode branch in perovskites because temperature-driven condensation of modes on this branch can describe many structural transitions which accompany ordering transitions of other types [1-3]. These soft modes circumscribe the BZ edges [Fig. 1(e)] within a very narrow window of energy set by the $M-R$ branch dispersion, with the $R$ point $\simeq 200 \mu \mathrm{eV}$ lower at all temperatures measured, consistent with an approach to the symmetry-lowered rhombohedral $\bar{R} 3 c$ structure, as observed in other $3 d \mathrm{BF}_{3}$ systems.

The rhombohedral $\bar{R} 3 c$ structure is related to the hightemperature cubic $P m \overline{3} m$ structure via a staggered octahedral tilt around the $\langle 111\rangle$ axis [Fig. 1(a) inset], corresponding to a frozen $R_{4}^{+}$lattice distortion [Fig. $1(\mathrm{~g})$ ]. The staggered 111
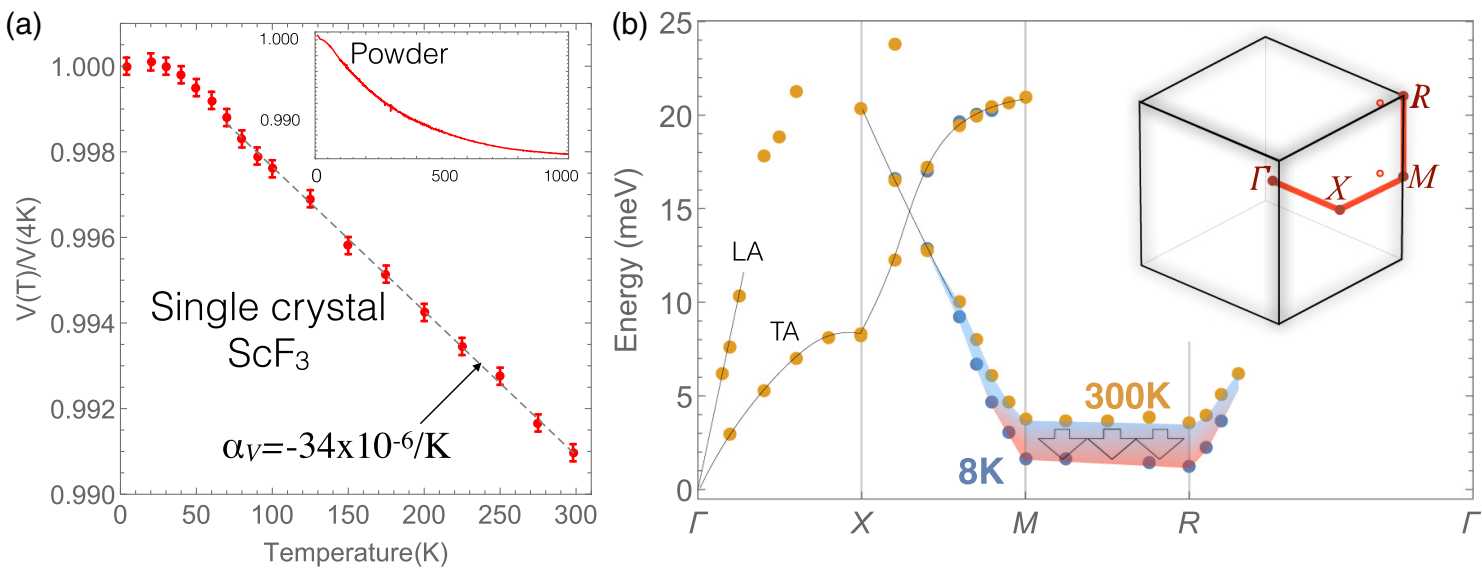

FIG. 2. (Color online) (a) Lattice volume as a function of temperature determined from tracking the Bragg peak $(3,0,0)$ in single-crystalline $\mathrm{ScF}_{3}$. Inset: The lattice volume over a larger thermal window determined on powder samples [7]. (b) Overview of the lattice mode dispersion at select momenta and energies. Strong branch softening along the $M-R$ cut is shown by arrows. The longitudinal (LA) and transverse (TA) acoustic branches are shown near $\Gamma$. Black solid lines are a guide to the eye. Red circles in the inset mark the momenta $R+\delta$ and $M+\epsilon$. 
octahedral rotation angle is therefore the order parameter of the rhombohedral phase. The free-energy curvature and origin of cubic-phase stabilization in trifluorides has been addressed in a body of theoretical and computational work aimed to generally understand perovskite structural phases. Density functional theory [20], molecular dynamics [21], and exact electrostatic energy considerations [22] of the insulating $\mathrm{AlF}_{3}$ prototype trifluoride suggest that Madelung energy including static electric dipole-dipole and induced dipole-induced dipole interactions compete to determine the stability of the cubic phase, and result in a low-temperature rhombohedral phase. It has been pointed out [22] that these competing influences could cancel identically for larger metal radii, marginally stabilizing the cubic phase for all temperatures, and it appears this limit is realized for $\mathrm{ScF}_{3}$. This suggestion is supported by related previous work using momentum-integrated timeof-flight neutron spectroscopy on bulk powder samples of $\mathrm{ScF}_{3}$ that showed a temperature dependence of high-energy $>10 \mathrm{meV}$ peaks in the neutron-weighted density of states and included calculations suggesting the curvature of the $R_{4}^{+}$mode is quartic in the tilt angle [23]. In what follows we present a momentum, energy, and temperature-resolved investigation of the approach to the quantum phase transition between the simple cubic and rhombohedral phases and its effects on the lattice dynamics of perovskite $\mathrm{ScF}_{3}$.

Figures 3(a) and 3(b) show the temperature-dependent spectra at $M(2.5,1.5,0)$ and $R(2.5,1.5,0.5)$ points, where the dynamical structure factor is particularly strong for the modes of Figs. 1(f) and 1(g). Clear soft mode behavior is observed concomitant with the emergence of a strong elastic peak, which onsets at $\sim 80 \mathrm{~K}$ and strengthens dramatically as the temperature is lowered. The elastic peak emergence is also observed at momenta away from the high-symmetry $M-R$ cut from data collected in an adjacent analyzer channel. Figures $3(\mathrm{c})$ and 3(d) show IXS data at $M+\epsilon(2.39,1.43,-0.02)$ and $R+\delta(2.39,1.43,0.45)$ momentum points, where the optical branch is much stiffer (5-6 meV) and displays a milder degree of softening, making the distinction between the elastic peak and soft mode phenomena more apparent in the raw data. In all cases, a transfer of spectral weight from the optical mode to the central peak feature is observed as the temperature is lowered.

All data were fit using a resolution-convoluted model for the dynamical structure factor, which includes a damped harmonic oscillator and a detailed balance condition to describe the lattice mode, and a resolution-limited elastic "central" peak, consistent with previous analyses of this phenomena in $\mathrm{SrTiO}_{3}$ and other materials [24] (see the Supplemental Material [15]). This analysis allows us to extract the soft mode frequency $\Omega$ and strength $A_{0}$ of the central peak. Figure 3(e) shows that the temperature dependence of the lowest soft mode frequency behaves approximately as $\Omega^{2} \propto\left(T-T_{c}\right)$, as expected from classical mean-field theories of structural phase transitions [25]. This simple extrapolation of the $R$ point soft mode frequency suggests that the phase transition occurs at $T_{c} \simeq$ $-39 \mathrm{~K}$, so it does not occur for any finite temperature, consistent with observations [7,9-11].

Central peaks and mode softenings were discovered in the context of the $110 \mathrm{~K}$ cubic-to-tetragonal transition of incipient ferroelectric $\mathrm{SrTiO}_{3}$ [24,26-28], but are commonly associated with an approach to a structural phase transition.
There, the elastic central peak has maximum strength at $T_{c}$ but begins to appear up to $25 \mathrm{~K}$ higher [24,28]. In the present case, we observe no maximum in elastic scattering at any finite temperature and the intensity continues to strengthen at the lowest temperatures measured $(3.8 \mathrm{~K})$. In $\mathrm{ScF}_{3}$, the effect of the central peak begins above $80 \mathrm{~K}$, suggesting that precursor effects occur as high as $120 \mathrm{~K}$ above our extrapolated transition temperature. The thermally robust central peak in $\mathrm{ScF}_{3}$ occurs in our strain-free, (naturally) isotopically pure, color center-free single crystals with a narrow $\left(0.002^{\circ}\right)$ mosaic. Interestingly, the elastic peak begins to strengthen at the same temperature scale at which the thermal expansion saturates, which is discussed further below.

Though central peaks have been observed in nearly all systems exhibiting structural phase transitions, their origins are still under debate [29]. While one class of theories has proposed that the central peak is the result of intrinsic nonlinear mechanisms such as the presence of solitons [30], another class of theories suggests that it is an extrinsic effect due to defects [29]. Based on the appearance of the central peak at a broad manifold of momenta throughout the Brillouin zone, we have observed localized excitations in the fluctuation spectrum below $100 \mathrm{~K}$. Below $T_{c}$ in the related material $\mathrm{TiF}_{3}$ [5], needlelike 111-oriented domains proliferate throughout the material, and we speculate that the localization of excited states may be a precursor effect related to the formation of the domain structure in the ordered state.

The $c-r$ structural transition can be stabilized at finite temperature through substitution of other metals (Al, Ti, Y) for $\mathrm{Sc}$, or in pure $\mathrm{ScF}_{3}$ through application of very low hydrostatic pressures [13,14] $(<0.2 \mathrm{GPa})$ at cryogenic temperatures. Remarkably, using the observed pressure dependence of the transition temperature $d T_{c} / d p \simeq 525 \mathrm{~K} / \mathrm{GPa}[7,13,14]$, and the putative transition temperature $T_{c} \simeq-39 \mathrm{~K}$, one can estimate that pressures as small as $740 \mathrm{bar}=0.074 \mathrm{GPa}$ would be sufficient to drive the transition upward to $0 \mathrm{~K}$, permitting a clean manner in which to observe a structural quantum phase transition. The sensitivity of the phase boundary to these external changes suggests that the nature of the cubic phase is delicate at low temperature and susceptible to even mild perturbations. Chemical substitutions of Sc by Ti [9], $\mathrm{Al}$ [11], and Y [10] have been reported and $T_{c}$ is presented in Fig. 1(a). The phase boundary in $\mathrm{Sc}_{1-x} \mathrm{Ti}_{x} \mathrm{~F}_{3}$ appears to linearly interpolate between the end members $\mathrm{ScF}_{3}$ and $\mathrm{TiF}_{3}$ with no maxima or minima at intermediate compositions, consistent with a picture where the substitution of wellmatched ionic radii ( $r_{\mathrm{Ti}}=0.670 \AA ; r_{\mathrm{Sc}}=0.745 \AA$ ) uniformly affects the potential landscape and gradually changes the phase stability.

On the other hand, substitution of ions with radii much smaller $\left(r_{\mathrm{Al}}=0.535 \AA\right)$ or much larger $\left(r_{\mathrm{Y}}=0.90 \AA\right)$ than Sc stabilizes the rhombohedral phase, suggesting the role of disorder is strong for these substitutions. We quantify the disorder in these solid solutions by using the variance of the $B$-site ion distribution $\sigma_{B}^{2}=\left\langle r_{B}-\left\langle r_{B}\right\rangle\right\rangle^{2}$ as determined from the nominal chemical formula in these solid solutions, and plot the disorder phase diagram in Fig. 4. The trend in $T_{c}\left(\sigma_{B}^{2}\right)$ is an example of the general case, wherein electronic (superconducting, magnetic) and structural (ferroelectric, ferroelastic) transitions have opposite sensitivities to disorder, as 

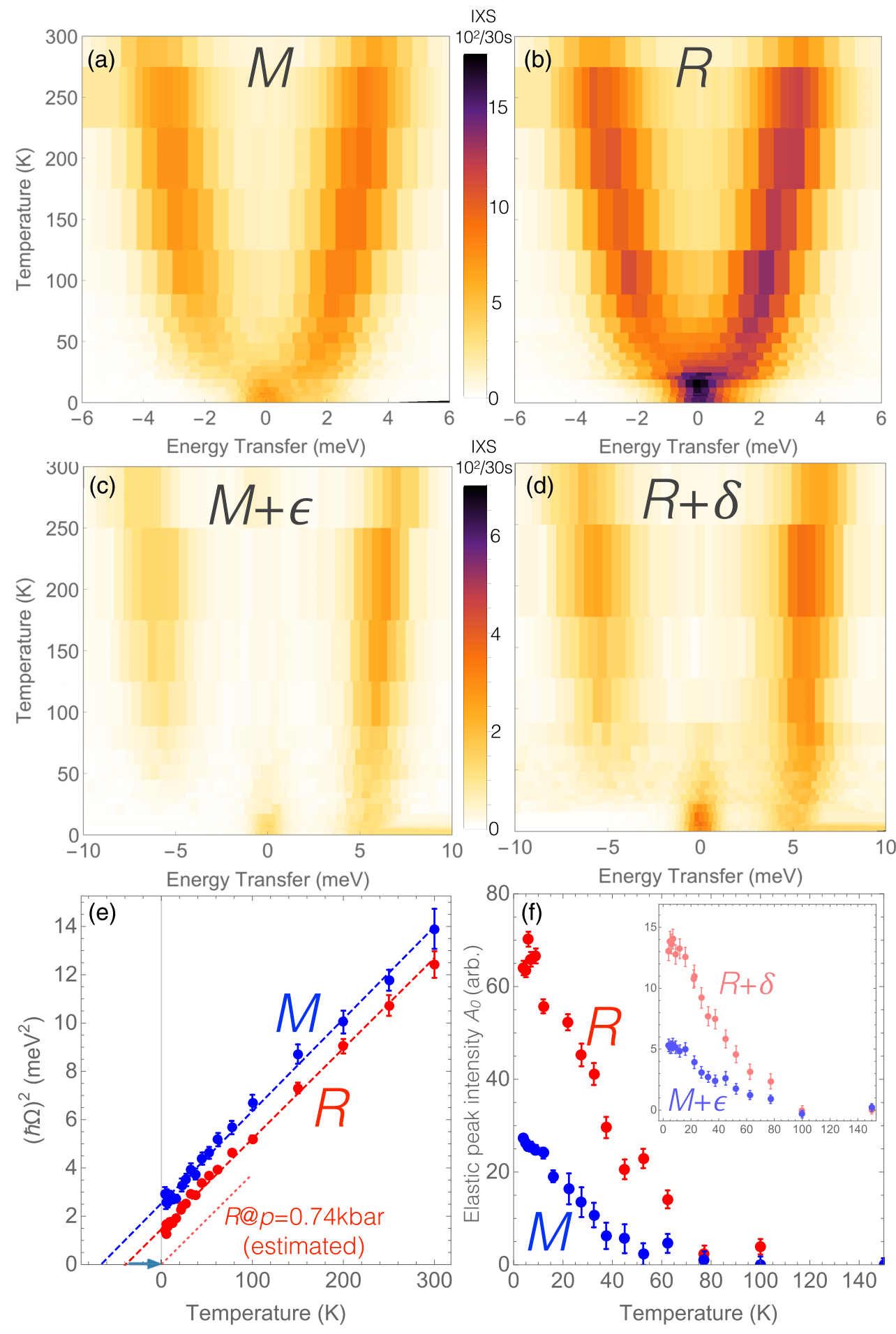

FIG. 3. (Color online) Color maps of the IXS signal at the (a) $M$, (b) $R$, (c) $M+\epsilon$, and (d) $R+\delta$ points in reciprocal space. Resolutionconvoluted fitting of the IXS data in (a)-(d) results in squared mode energies (e) and elastic peak intensity (f). Dashed lines in (e) show the extrapolation of the mode frequencies from classical mean-field theory. Dotted lines show the expected effect of a small $74 \mathrm{MPa}$ pressure on the $R$ point mode energy, suggesting this small pressure is necessary to pass through the QPT.

determined through studies of the $A$-site variance effect on phase stability $[31,32]$. The latter case, relevant here, occurs because local strain associated with substitution has the effect of disrupting long-range propagation of strain, enhancing the stability of the lowered-symmetry phase. Generalizing these trends to $B$-site disorder near the composition $\mathrm{ScF}_{3}$, we separately fit the transition temperatures $T_{c}(x)=T_{c}^{0}-p_{1} \sigma_{B}^{2}$ to the doped compositions of $\mathrm{Al}$ and $\mathrm{Y}$ substitution and find that extrapolating transition temperatures of the doped compositions to the ideal case $\sigma_{B}^{2}=0$ gives roughly similar 


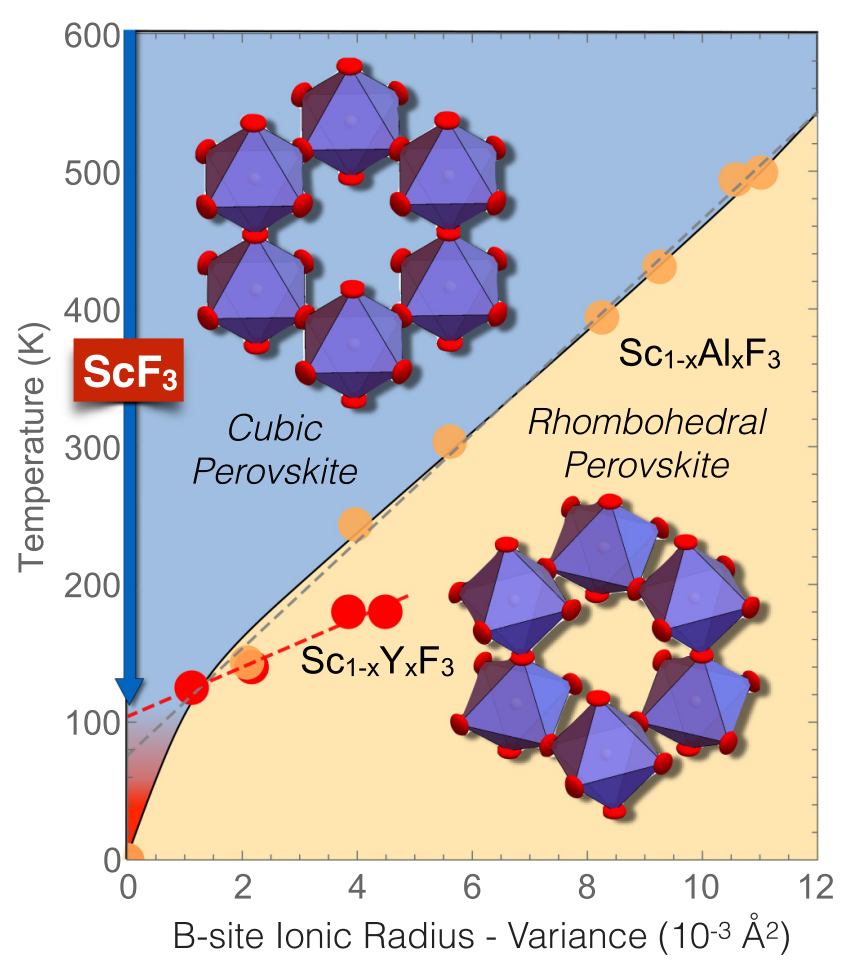

FIG. 4. (Color online) Disorder phase diagram for $\mathrm{ScF}_{3}$. Compositional disorder is quantified via the $B$-site variance $\sigma_{B}^{2}=\left\langle r_{B}-\right.$ $\left.\left\langle r_{B}\right\rangle\right\rangle^{2}$, which has the effect of increasing the stability of the lowersymmetry, rhombohedral structural phase. Red and orange symbols show the effect of $\mathrm{Y}$ and $\mathrm{Al}$ substitution, respectively. Dotted lines indicate a linear fit to the $T_{c}\left(\sigma_{B}^{2}\right)$, not including the pure sample $\mathrm{ScF}_{3}$. The vertical axis intercept reveals a temperature scale $\simeq 80-100 \mathrm{~K}$, where central peak elastic scattering and thermal expansion saturation are also observed in pure $\mathrm{ScF}_{3}$.

$T_{c}^{0}=75 \mathrm{~K}(\mathrm{Al})$ and $104 \mathrm{~K}(\mathrm{Y})$, which is near the temperature scale where we observe a strong elastic peak emergence [Fig. 3(f)] and the thermal expansion coefficient departs from linear dependence on temperature [Fig. 2(a)]. Empirically, $T_{c}<0.4 \mathrm{~K}$ is observed for pure $\mathrm{ScF}_{3}$, suggesting a strong deviation from previous studies [31,32] and qualitatively different physics in the pure limit.
The $\mathrm{ScF}_{6}$ octahedra in pure $\mathrm{ScF}_{3}$ move collectively in a state of strong orientational fluctuation [22,23], the strength of which can be estimated from powder refinement of the anisotropic thermal parameter $U_{33}$, associated with transverse F motion. The orientational fluctuations are significant [7], approaching $2 \sqrt{U_{33}} / a \simeq 9.2^{\circ}$ full width at half maximum (FWHM) at room temperature, significantly larger than the isostructural oxide $\mathrm{ReO}_{3}$ [33]. In this regime, the influence of long-ranged strain draws in the lattice, leading to a robust and sizable NTE effect. At elevated temperatures, long-range strain fields imposed by local constraints propagate over much shorter distances, and the fluctuation spectrum lacks a central peak and appears more conventional. The long-range strain effects we have observed are likely present in the physics of structural transitions in perovskite and other structural classes, but are particularly enhanced in $\mathrm{ScF}_{3}$ at low temperature.

In conclusion, we have observed signatures of an approach to a structural phase transition occurring close to zero temperature and pressure in the strong negative thermal expansion materials $\mathrm{ScF}_{3}$. We have identified the zoneboundary soft mode branch associated with an incipient structural transition in $\mathrm{ScF}_{3}$, together with a thermally robust, resolution-limited central peak. Analysis of the lattice instability suggests that weak pressures are sufficient to induce the $c-r$ transition very near zero temperature. These observations indicate that $\mathrm{ScF}_{3}$ is a candidate material to explore the effects of quantum mechanics on central peaks and structural phases [36,37].

The authors also acknowledge the valuable conversations with Joe Budnick and Gabe Aeppli. Work at the University of Connecticut is supported by National Science Foundation Award No. DMR-1506825. Work at the University of Costa Rica is supported by Vicerrectoría de Investigación under Project No. 816-B5-220, and work at Argonne National Laboratory is supported by the US Department of Energy, Office of Basic Energy Sciences under Contract No. DEAC02-06CH11357. R.T.B. acknowledges support from the Yale Prize Postdoctoral Fellowship. The construction of HERIX was partially supported by the NSF under Grant No. DMR-0115852.
[1] M. E. Lines and A. M. Glass, Principles and Applications of Ferroelectrics and Related Materials (Clarendon, Oxford, U.K., 1979).

[2] Physics of Transition Metal Oxides, edited by S. Maekawa, T. Tohyama, S. Barnes, S. Ishihara, W. Koshibae, and G. Khaliullin (Springer, Berlin, 2004).

[3] N. A. Benedek and C. J. Fennie, J. Phys. Chem. C 117, 13339 (2013).

[4] $\mathrm{MnF}_{3}$ forms a triclinic structure [34], likely resultant from unusually strong magnetic and Jahn-Teller effects [35]. We have not included this in the phase diagram.

[5] A. Mogus-Milankovic, J. Ravez, J. Chaminade, and P. Hagenmuller, Mater. Res. Bull. 20, 9 (1985).

[6] C. P. Romao, C. R. Morelock, M. B. Johnson, J. W. Zwanziger, A. P. Wilkinson, and M. A. White, J. Mater. Sci. 50, 3409 (2015).

[7] B. K. Greve, K. L. Martin, P. L. Lee, P. J. Chupas, K. W.
Chapman, and A. P. Wilkinson, J. Am. Chem. Soc. 132, 15496 (2010).

[8] P. Daniel, A. Bulou, M. Rousseau, J. Nouet, and M. Leblanc, Phys. Rev. B 42, 10545 (1990).

[9] C. R. Morelock, L. C. Gallington, and A. P. Wilkinson, Chem. Mater. 26, 1936 (2014).

[10] C. R. Morelock, B. K. Greve, L. C. Gallington, K. W. Chapman, and A. P. Wilkinson, J. Appl. Phys. 114, 213501 (2013).

[11] C. R. Morelock, L. C. Gallington, and A. P. Wilkinson, J. Solid State Chem. 222, 96 (2015).

[12] A. H. Said, H. Sinn, and R. Divan, J. Synchrotron Radiat. 18, 492 (2011).

[13] K. S. Aleksandrov, V. N. Voronov, A. N. Vtyurin, A. S. Krylov, M. S. Molokeev, M. S. Pavlovski, S. V. Goryanov, A. Y. Likhacheva, and A. I. Ancharov, Phys. Solid State 51, 810 (2009). 
[14] K. S. Aleksandrov, N. V. Voronov, A. N. Vtyurin, A. S. Krylov, M. S. Molokeev, A. S. Oreshonkov, S. V. Goryainov, A. Y. Likhacheva, and A. I. Ancharov, Phys. Solid State 53, 564 (2011).

[15] See Supplemental Material at http://link.aps.org/supplemental/ 10.1103/PhysRevB.92.134101 for further details regarding the experiment, single crystal growth and data analysis.

[16] C. Pantea, A. Migliori, P. B. Littlewood, Y. Zhao, H. Ledbetter, J. C. Lashley, T. Kimura, J. Van Duijn, and G. R. Kowach, Phys. Rev. B 73, 214118 (2006).

[17] J. N. Grima, V. Zammit, and R. Gatt, Xjenza 11, 17 (2006).

[18] C. Lind, Materials 5, 1125 (2012).

[19] K. Takenaka, Sci. Technol. Adv. Mater. 13, 013001 (2012).

[20] L. L. Boyer, M. J. Mehl, and D. Finkenstadt, Phys. Rev. B 75, 132103 (2007).

[21] S. Chaudhuri, P. J. Chupas, M. Wilson, P. Madden, and C. P. Grey, J. Phys. Chem. B 108, 3437 (2004).

[22] P. B. Allen, Y.-R. Chen, S. Chaudhuri, and C. P. Grey, Phys. Rev. B 73, 172102 (2006).

[23] C. W. Li, X. Tang, J. A. Muñoz, J. B. Keith, S. J. Tracy, D. L. Abernathy, and B. Fultz, Phys. Rev. Lett. 107, 195504 (2011).

[24] S. Shapiro, J. Axe, G. Shirane, and T. Riste, Phys. Rev. B 6, 4332 (1972).

[25] R. Cowley, Adv. Phys. 29, 1 (1980).
[26] T. Riste, E. Samuelsen, K. Otnes, and J. Feder, Solid State Commun. 9, 1455 (1971).

[27] K. A. Muller and T. W. Kool, Properties of Perovskites and Other Oxides (World Scientific, Singapore, 2010).

[28] M. Kisiel, F. Pellegrini, G. Santoro, M. Samadashvili, R. Pawlak, A. Benassi, U. Gysin, R. Buzio, A. Gerbi, E. Meyer et al., Phys. Rev. Lett. 115, 046101 (2015).

[29] R. Cowley and S. M. Shapiro, J. Phys. Soc. Jpn. 75, 111001 (2006).

[30] J. A. Krumhansl and J. R. Schrieffer, Phys. Rev. B 11, 3535 (1975).

[31] L. M. Rodriguez-Martinez and J. P. Attfield, Phys. Rev. B 54, R15622 (1996).

[32] J. Attfield, Int. J. Inorg. Mater. 3, 1147 (2001).

[33] E. E. Rodriguez, A. Llobet, T. Proffen, B. C. Melot, R. Seshadri, P. B. Littlewood, and A. K. Cheetham, J. Appl. Phys. 105, 114901 (2009).

[34] M. A. Hepworth and K. H. Jack, Acta Crystallogr. 10, 345 (1957).

[35] B. A. Hunter, B. J. Kennedy, and T. Vogt, Phys. Rev. B 69, 020410 (2004).

[36] M. Zacharias, I. Paul, and M. Garst, Phys. Rev. Lett. 115, 025703 (2015).

[37] S. E. Rowley, L. J. Spalek, R. P. Smith, M. P. M. Dean, M. Itoh, J. F. Scott, G. G. Lonzarich, and S. S. Saxena, Nature Physics 10, 367 (2014). 